\title{
Simulation of Ectopic Activity Onset in Border Zones Between Normal and Damaged Myocardium with Minimal Ionic Models
}

\author{
Maxim Ryzhii ${ }^{1}$, Elena Ryzhii ${ }^{2}$ \\ ${ }^{1}$ University of Aizu, Aizu-Wakamatsu, Japan \\ ${ }^{2}$ Fukushima Medical University, Fukushima, Japan
}

\begin{abstract}
We use popular minimal ionic models - two-variable Mitchell-Schaeffer model and its modification (CorradoNiederer), and four-variable Bueno-Orovio-Cherry-Fenton model to investigate the possibility to obtain an onset of ectopic activity in border zones between normal and damaged myocardial tissue. For the simulations of damaged tissue we use bistable and ultra-long action potentials of cardiomyocytes. We also present a brief nonlinear analysis of the conditions required for the ectopic activity onset. We conclude that the appearance of the ectopic excitation and its location depend on each model propensity of spontaneous depolarization.
\end{abstract}

\section{Introduction}

Ectopic activity, which is an abnormal excitation in cardiac tissue, is one of the triggers for dangerous arrhythmia initiation. Recently, the paradoxical onset of ectopic sources in the border zones between normal and damaged myocardium was reported in experiments with optogenetically modified monolayers of cardiomyocytes, accompanied by computer simulations and theoretical explanations [1]. The study claims that primary ectopic excitation takes place at the areas of maximal curvature, such as corners of damaged tissue regions (DR) with ultra-long action potentials (AP), in which, as stipulated in the paper, the stimulating coupling currents between cells do not correspond to classical source-sink relationship for successful excitation of the neighboring tissue. This effect was reported for the first time and seems very interesting for further investigations. In their computer simulations the authors used some simple phenomenological models and complex ion-channel models for ventricular myocytes adapted to represent pathological properties of the DR.

In this study, we use two-variable Mitchell-Schaeffer (MS) model [2], its modification by Corrado and Niederer (CN) [3], and four-variable Bueno-Orovio-Cherry-Fenton (BOCF) model [4] to investigate the possibility to obtain the paradoxical effect mentioned above in these popular minimal ionic models. We also reproduce as a reference the results for simulations with FitzHugh-Nagumo (FHN) model [5] demonstrated in [1].

We present a brief nonlinear analysis and comparison of properties of the above models and attempt to clarify the mechanism of the ectopic activity initiation in the border zones between normal and damaged cardiac tissue. Our study can also provide some additional insight into the origin of triggers of dangerous cardiac arrhythmias.

\section{Models}

The simulation model setup represents a square area of normal tissue with small square DR in the center. Excitation front propagates from the right side of the normal area and, upon passing through the DR, eventually spontaneous depolarizations may appear near the borders of the normal and damaged tissue due to the electrotonic interactions in the presence of large gradients of the trans-membrane potentials. Similar to [1], we implemented two variants of damaged tissue - with bistable (subscript B) and ultra-long (subscript L) APs for all models considered.

First, as a reference we reproduced some of the simulation results from [1] using the FHN model:

$$
\begin{gathered}
\partial_{t} u=u(u-a)(1-u)-v+I_{s t i m}, \\
\partial_{t} v=\epsilon_{F H N}(u-\gamma v),
\end{gathered}
$$

where $u$ is the trans-membrane potential, $v$ is the gate variable, $I_{\text {stim }}=D\left(\partial_{x}^{2} u+\partial_{y}^{2} u\right)$ is the diffusion term, and $D$ is the diffusion coefficient. The parameters were taken similar to that in [1]: $a=0.13$ for all AP variants, for normal AP $-\epsilon_{F H N}=0.004$ and $\gamma=1.5$, for bistable $\mathrm{AP} \epsilon_{F H N}=0.004$ and $\gamma=13$, and for long AP $\epsilon_{F H N}=0.00005$ and $\gamma=3.12$.

The two-variable MS model and its $\mathrm{CN}$ modification were represented by the following common set of equations:

$\partial_{t} u=h u\left(u-u_{g} c\right)(1-u) / \tau_{\text {in }}-(1-h c) u / \tau_{\text {out }}+I_{\text {stim }}$, 

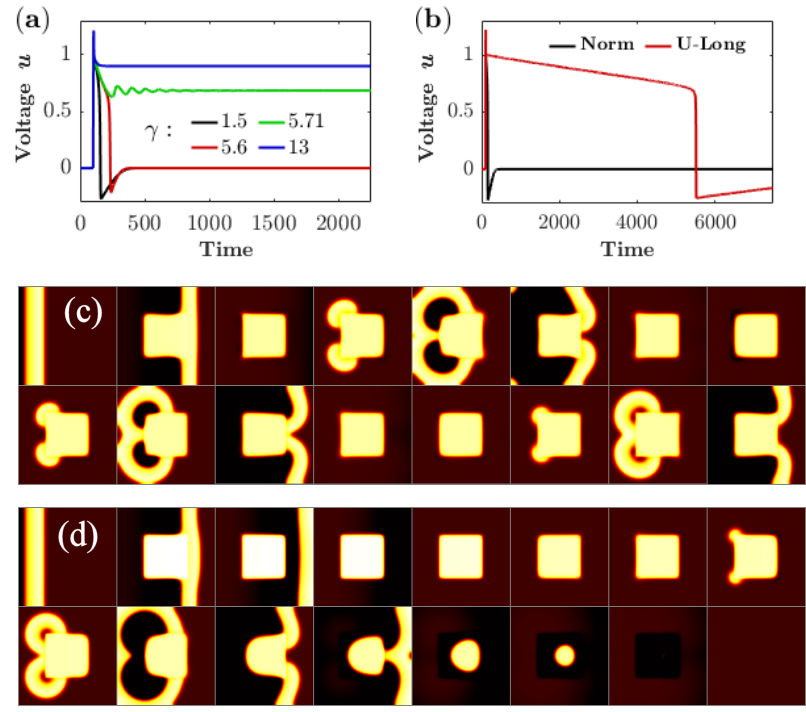

Figure 1. The FHN model: Bistable (a) and ultra-long (b) DR APs of uncoupled cells, and snapshots of ectopic activity with bistable (c) and ultra-long (d) AP variants. In (a) the APs calculated with different values of $\gamma$ are shown.

Table 1. Parameters for the MS, CN, and CN-MS models.

\begin{tabular}{lcccccc}
\hline \hline Model & $\tau_{\text {in }}$ & $\tau_{\text {out }}$ & $\tau_{\text {open }}$ & $\tau_{\text {close }}$ & $u_{g}$ & $\eta$ \\
\hline Normal & 0.3 & 6 & 120 & 150 & 0.13 & 0.001 \\
\hline MS $_{B}$ & 0.3 & 6 & 120 & 150 & 0.60 & 0.001 \\
$\mathrm{MS}_{L}$ & 0.6 & 48 & 720 & 1200 & 0.13 & 0.001 \\
$\mathrm{CN}_{B}$ & 0.6 & 24 & 240 & 600 & 0.13 & 0.60 \\
$\mathrm{CN}_{L}$ & 0.6 & 48 & 720 & 1200 & 0.13 & 0.001 \\
$\mathrm{CN}-\mathrm{MS}_{B}$ & 0.6 & 12 & 240 & 300 & 0.40 & 0.30 \\
$\mathrm{CN}-\mathrm{MS}_{L}$ & 0.6 & 24 & 240 & 1800 & 0.13 & 0.001 \\
\hline \hline
\end{tabular}

$$
\begin{gathered}
\partial_{t} h=\epsilon_{M S}\left(h_{\infty}-h\right), \\
\epsilon_{M S}=1 / \tau_{\text {close }}+h_{\infty}\left(\tau_{\text {close }}-\tau_{\text {open }}\right) /\left(\tau_{\text {close }} \tau_{\text {open }}\right), \\
h_{\infty}=\left(1-\tanh \left(\left(u-u_{g}\right) / \eta\right)\right) / 2,
\end{gathered}
$$

where $h$ is the gate variable for the inward current, $u_{g}$ is the activation threshold potential, $\tau_{i n}, \tau_{\text {out }}, \tau_{\text {open }}$, and $\tau_{\text {close }}$ are the time constants affecting the corresponding characteristic phases of the trans-membrane potential evolution.

Together with $u_{g}$, the parameter $\eta$ is responsible for the cell spontaneous excitation properties. When parameter $\eta$ is small enough $(\eta \leq 0.001)$ the formulation (4) for $\partial_{t} h$ closely corresponds to the original piece-wise function [2]. Here we use generalized formulation from [6] for $\partial_{t} h(a=0$ and $\lambda=0)$ instead of original piece-wise function. To allow switching between the models we introduce the parameter $c$, with $c=0$ for the MS and $c=1$ for the $\mathrm{CN}$ models, respectively.

As the $\mathrm{CN}$ model has been proved to be robust to the pacemaker behavior [3], it was interesting to check its per-
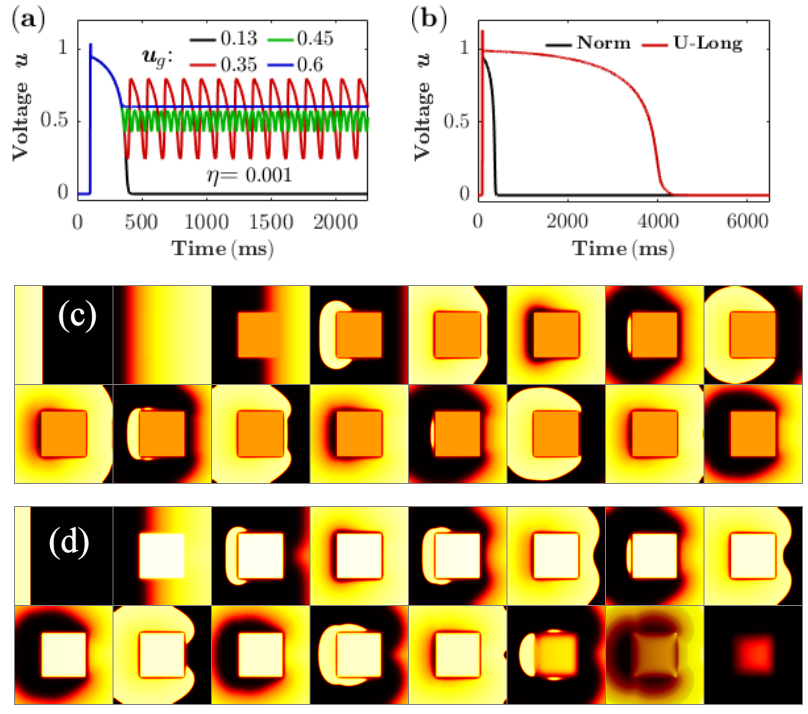

Figure 2. The MS model: Bistable (a) and ultra-long (b) DR APs of uncoupled cells, and snapshots of ectopic activity with bistable (c) and ultra-long (d) AP variants. In (a) the APs calculated with different values of $u_{g}$ are shown.

formance in the present simulation setup. We also tested combined CN-MS model where the $\mathrm{CN}$ model represents normal tissue, and the MS model - the DR.

The parameters for normal tissue, MS bistable $\left(\mathrm{MS}_{B}\right)$, MS ultra-long $\left(\mathrm{MS}_{L}\right), \mathrm{CN}$ bistable $\left(\mathrm{CN}_{B}\right), \mathrm{CN}$ ultra-long $\left(\mathrm{CN}_{L}\right), \mathrm{CN}-\mathrm{MS}$ bistable $\left(\mathrm{CN}-\mathrm{MS}_{B}\right)$, and CN-MS ultralong $\left(\mathrm{CN}-\mathrm{MS}_{L}\right)$ model variants are presented in Table 1 .

For the BOCF model we used original formulation with the data for epicardial myocytes [4], in which we changed the values of $\tau_{w}^{+}$from $200 \mathrm{~ms}$ to $5000 \mathrm{~ms}$ and $k_{s o}$ from 2.0458 to 2.4 in order to obtain ultra-long AP in the DR. It was difficult to implement bistable AP version owing to the relative complexity of the BOCF model, so we had to restrict ourselves to the ultra-long AP variant only.

All simulations were performed in 512 x 512 normal region, and $260 \times 260 \mathrm{DR}$ with time step $\Delta t=0.02$ $\mathrm{ms}$, spatial step $\Delta x=0.0625 \mathrm{~mm}$, and diffusion coefficient $D=0.0154 \mathrm{~mm}^{2} / \mathrm{ms}$ (for the FHN model all parameters were dimensionless). Numerical integration was performed with forward Euler scheme.

\section{Results and discussion}

The bistable and ultra-long APs of uncoupled model cells and the corresponding snapshots of the simulated excitation sequences for the models are presented in Figures 1-5. The results shown in Figure 1 for the FHN model are practically similar to the ones in [1]. The ectopic onset for both bistable and ultra-long APs [Figures 1(a) and 1(b)] took place only from corners of the DR. The activity con- 

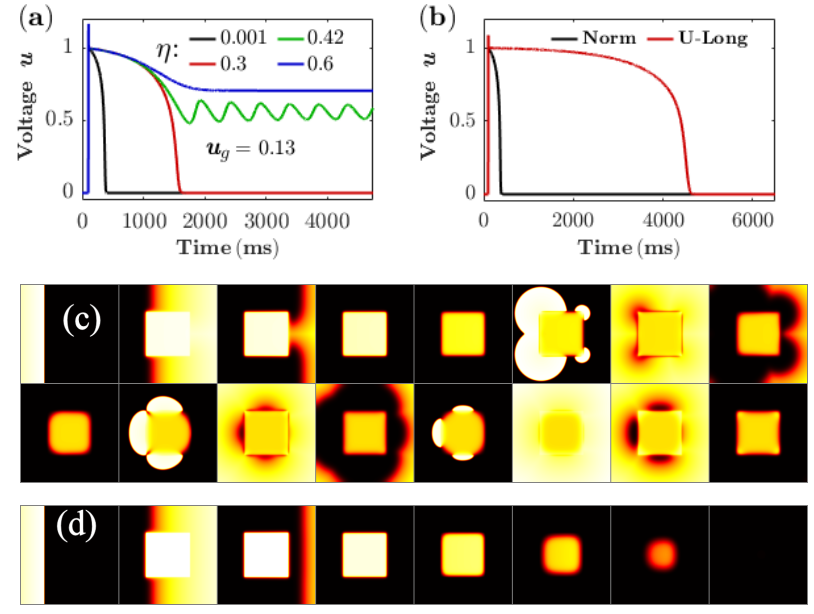

Figure 3. The CN model: Bistable (a) and ultra-long (b) DR APs of uncoupled cells, and snapshots of ectopic activity with bistable AP (c) and excitation sequence with ultra-long AP (d) variants. In (a) the APs calculated with different values of $\eta$ are shown.

tinued for infinitely long period of time in the bistable case and ceased after one cycle for the $\mathrm{FHN}_{L}$ [see Figures 1(c) and $1(\mathrm{~d})$, respectively].

Choosing the proper value of $u_{g}$ for stable AP in the $\mathrm{MS}_{B}$ model, we had to avoid the region where the AP becomes oscillatory. Such behavior of the original MS model has been mentioned in [3] resulting in proposal of its robust modification $(\mathrm{CN})$. The isolated APs calculated for $\mathrm{MS}_{B}$ with different values of $u_{g}$ are shown in Figure 2(a). The oscillating behavior appears in the region $0.3<u_{g}<0.5$, so $u_{g}=0.6$ was selected for further simulations. For both $\mathrm{MS}_{B}$ and $\mathrm{MS}_{L}$ variants, the spontaneous frequent excitations originated from the sides of the DR [Figures 2(a) and 2(b)]. We did not observe the excitation from the corners of DR in both MS models, which can be explained by premature depolarization from the sides. Such a behavior can be attributed to the general instability of the original MS model [7].

For the $\mathrm{CN}$ model, we could obtain non-oscillating bistable AP in DR only by setting the value of $\eta=0.6$ [Figure 3(a)]. The $\mathrm{CN}_{B}$ variant demonstrated both corner and side spontaneous excitations [Figure 3(c)], while no onset of ectopic sources was observed in the $\mathrm{CN}_{L}$ case [Figure 3(d)].

The combined CN-MS model was considered in order to enhance stability in the normal tissue region. For the $\mathrm{CN}^{-\mathrm{MS}_{B}}$ variant we set $\eta=0.3$ and $u_{g}=0.4$ to get nonoscillating bistable isolated APs in the DR [Figure 4(a)]. Ectopic sources appeared at corners only for the $\mathrm{CN}-\mathrm{MS}_{B}$, and at both corners and sides for the CN-MS ${ }_{L}$. Permanent quasi-chaotic self-excitation patterns inside the DR were
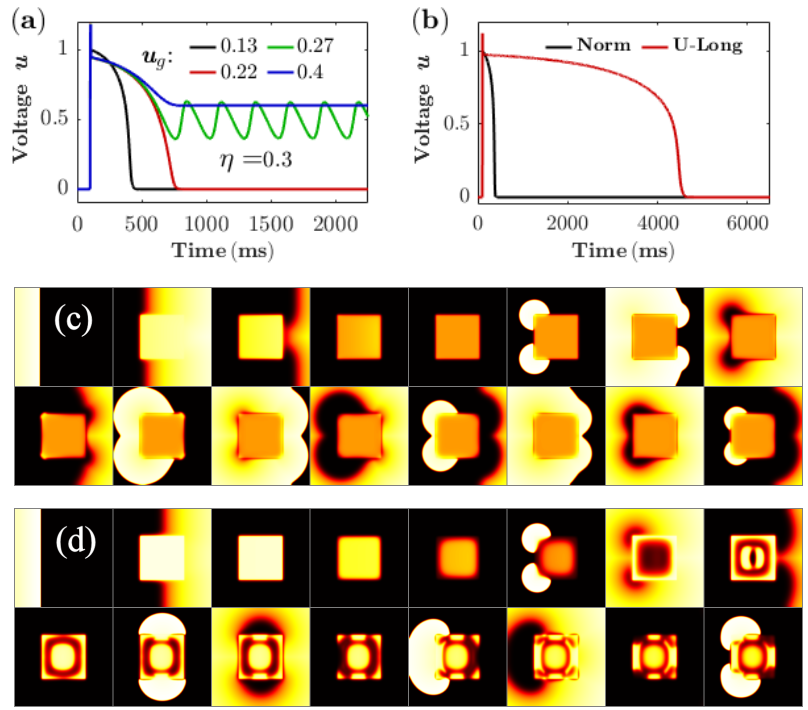

Figure 4. The CN-MS model: Bistable (a) and ultra-long (b) DR APs of uncoupled cells, and snapshots of ectopic activity with bistable (c) and ultra-long (d) AP variants. In (a) the APs calculated with different values of $u_{g}$ are shown.

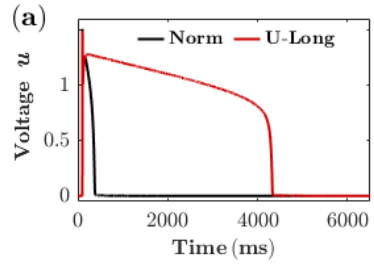

(b)

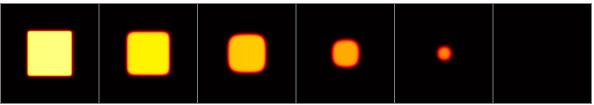

Figure 5. The $\mathrm{BOCF}_{L}$ model: Ultra-long DR AP of uncoupled cells (a), and snapshots of excitation sequence (b).

observed for the CN-MS $\mathrm{M}_{L}$ model as seen in Figure 4(d).

Finally, we were unable to get any spontaneous excitations for the BOCF model with ultra-long AP in the DR (Figure 5). As the BOCF model was derived from Fenton-Karma model [8], which had been proved to have only a unique and stable non-resting steady state [9], we can suggest that the former model has the same properties and wherefore does not demonstrate spontaneous excitation ability.

The comparison of the simulation results for all models and their variants is presented in Table 2 .

Figure 6 shows the phase portraits of the uncoupled bistable APs in the $\mathrm{FHN}_{B}, \mathrm{MS}_{B}, \mathrm{CN}_{B}$, and $\mathrm{CN}-\mathrm{MS}_{B}$ models corresponding to Figures 1(a), 2(a), 3(a), and 4(a), respectively. The onset of the pacemaker activity observed in the simulations can be explained as follows. An increase 
Table 2. Comparison of excitation types for the considered models and their variants.

\begin{tabular}{lccccccccc}
\hline \hline Model & $\mathrm{FHN}_{B}$ & $\mathrm{FHN}_{L}$ & $\mathrm{MS}_{B}$ & $\mathrm{MS}_{L}$ & $\mathrm{CN}_{B}$ & $\mathrm{CN}_{L}$ & $\mathrm{CN}^{-M_{B}}$ & $\mathrm{CN}-M S_{L}$ & $\mathrm{BOCF}_{L}$ \\
\hline $\begin{array}{l}\text { Corner excitation } \\
\text { Side excitation }\end{array}$ & + & + & & & + & & + & + & \\
\hline \hline
\end{tabular}
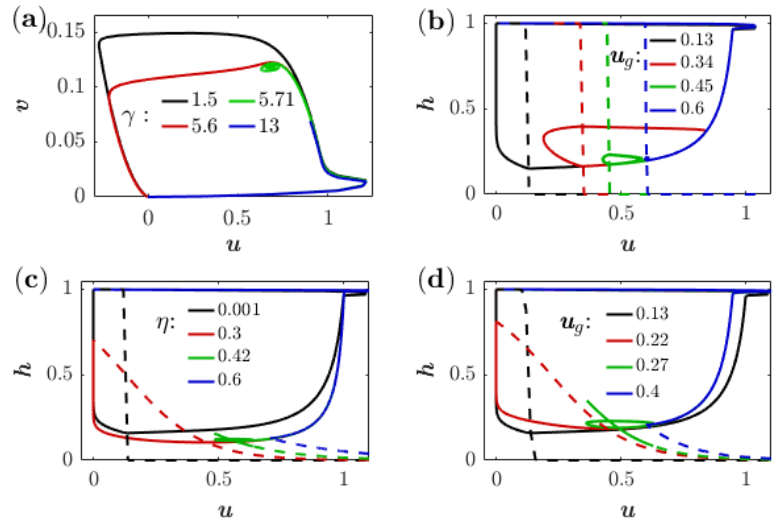

Figure 6. Phase portraits of uncoupled APs for the models: (a) - $\mathrm{FHN}_{B}$ with different $\gamma$, (b) - $\mathrm{MS}_{B}$ with different $u_{g}$, (c) $-\mathrm{CN}_{B}$ with different $\eta$, and (d) - CN-MS ${ }_{B}$ with different $u_{g}$. Dashed lines correspond to $h_{\infty}$.

in the parameter $\eta$ inclines the $h_{\infty}$ curve from vertical and, in combination with intercellular coupling, may lead to the appearance of limit cycle in the cells at the DR boundary [Figures 6(b-d)]. This effect is known as the effect of induced pacemaking [10] caused by electrotonic interactions between cells with the significant difference in the transmembrane potentials [11] or by additional factors, such as the mechano-electrical coupling [12].

A short video with the results can be found at [13].

\section{Conclusions}

In this work we studied initiation of the spontaneous ectopic activity in the boundary of damaged area using three popular minimal ionic models. The damaged regions were represented by bistable and ultra-long APs. We can conclude that the appearance of the ectopic excitation depends on the model properties, in particular, the tendency to pacemaker activity. In contrast to [1], we observed the onset of ectopic sources not only at the corners, but also at the sides of DR, therefore we cannot support the statement that its occurrence depends on the curvature of the DR boundary.

\section{Acknowledgments}

The work was supported by JSPS KAKENHI Grant No. 20K12046.

\section{References}

[1] Teplenin AS, Dierckx H, DeVries AAF, Pijnappels DA, Panfilov AV. Paradoxical onset of arrhythmic waves from depolarized areas in cardiac tissue due to curvaturedependent instability. Phys Rev X 2018;8:Art. no. 021077.

[2] Mitchell CC, Schaeffer DG. A two-current model for the dynamics of cardiac membrane. Bull Math Biol 2003;65:767-793.

[3] Corrado C, Niederer SA. A two-variable model robust to pacemaker behaviour for the dynamics of the cardiac action potential. Math Biosci 2016;281:46-54.

[4] Bueno-Orovio A, Cherry EM, Fenton FH. Minimal model for human ventricular action potentials in tissue. J Theor Biol 2008;253:544-560.

[5] FitzHugh RA. Impulses and physiological states in theoretical models of nerve membrane. Biophys J 1961;1(6):445466.

[6] Djabella K, Landau M, Sorine M. A two-variable model of cardiac action potential with controlled pacemaker activity and ionic current interpretation. In 46th IEEE Conf on Decision and Control 2007. IEEE 2007; 5186-5191.

[7] Corrado C, Whitaker J, Chubb H, William S, Wright M, Gill J, O'Neill MD, Niederer SA. Personalized models of human atrial electrophysiology derived from endocardial electrograms. IEEE Trans Biomed Eng 2017;64(4):735742.

[8] Fenton F, Karma A. Vortex dynamics in three-dimensional continuous myocardium with fiber rotation: Filament instability and fibrillation. Chaos 1998;8(1):20-47.

[9] Jacquemet V. Steady-state solutions in mathematical models of atrial cell electrophysiology and their stability. Math Biosci 2007;208:241-269.

[10] Antzelevitch C, Burashnikov A. Overview of basic mechanisms of cardiac arrhythmia. Card Electrophysiol Clin 2011;3:23-45.

[11] Jacquemet V. Pacemaker activity resulting from the coupling with nonexcitable cells. Phys Rev E 2006;74:Art. no. 011908.

[12] Panfilov AV, Keldermann RH, Nash MP. Self-organized pacemakers in a coupled reaction-diffusion-mechanics system. Phys Rev Lett 2005;95:Art. no. 258104.

[13] www.u-aizu.ac.jp/labs/cs-csm/mr/video/CINC2020_142.mp4.

Address for correspondence:

Maxim Ryzhii

University of Aizu

Aizu-Wakamatsu 965-8580, Japan

m-ryzhii @ u-aizu.ac.jp 